\title{
The formation of Zamboanga Chabacano language sentences
}

Pahulaya, Vilma L. $\square$

Western Mindanao State University, Philippines (Vilma.pahulaya05@gmail.com)

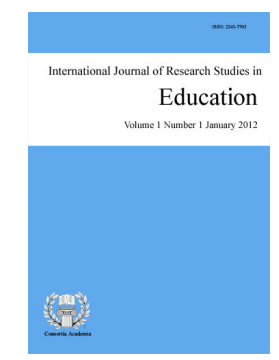

ISSN: 2243-7703 Online ISSN: 2243-7711

OPEN ACCESS

\section{Abstract}

The purpose of this study is to describe and evaluate the sentences in the Zamboanga Chabacano language in the city of Zamboanga. Qualitative design was used in this study. Descriptive analysis was used to analyze the sentences in the Zamboanga Chabacano language. Purposive sampling was used in selecting the respondents in this study. The instrument of this study was actual conversations of the respondents. These conversations come from seven domains; at home, at school, at church, at the bus terminal, at the market, at the internet Café and at the mall. It is confirmed and validated that what the participants in the conversation are Zamboanga Chabacano language. The focus of the analysis is on the character of the sentence, the word structure in the sentence construction and the markers within the sentence. The study found that the part of the sentences of Zamboanga Chabacano consist of subject and predicate. There are two sentence structures; the ordinary and unusual order. Whether the subject or predicate precedes the sentence does not change meaning of the said sentence. Except for the pronoun, the subject of the sentence is always marked. The predicate has no marker within the sentence. Conjunctive verbs used in a sentence are only consonants and prepositions. There is no proper Zamboanga Chabacano language. The punctuation marks used in a sentence are the preposition, preposition and the punctuation mark of the verb. Verb compliments are moved to different position within the sentence; front, middle, or rear changing the meaning of the said sentence.

Keywords: Zamboanga Chabacano language; sentences; characteristics; parts and order; structure words; conjunction and marker 


\section{The formation of Zamboanga Chabacano language sentences}

\section{Introduksyon}

Kung ang tao ang sinasabing pinakamagandang nilikha ng Diyos sa mundo, ang wika naman ang pinakamaganda Niyang regalo sa tao. Ang wika ay salamin ng kaisipan at saloobin ng tao, kung saan ito ang pangunahing kasangkapan sa komunikasyon. Hindi lamang layunin ng pakikipagtalastasan na maipahayag ang mensahe sa kausap, kundi ito ay maihatid din nang malinaw, wasto at kalugud-lugod, maging ito ay sa paraang pasalita o pasulat. Mahalaga ang patuloy na pag-aaral sa wika ng bawat lipon ng tao. Wika ang nagbibigay ng pagkakakilanlan ng tao sa lipunan. Mahalaga ang pagpapayabong ng wika ninuman. Sa ganoon nagkakaroon ng daan ng pagkalat ng isang wika at napanatili itong buhay at tuluyan nang napipreserba sa halip na isang patay na wika o dead language. Katulad na lamang sa wikang Zamboanga Chabacano na sa kabila na sinasabi ng iba na ang wikang Chabacano ay walang gramatika.

Nais ng kasalukuyang pag-aaral na ipakilala ang wikang Chabacano sa lungsod ng Zamboanga bilang isang natatanging wika sa pamamagitan ng paglalarawan ng estrukturang gramatikal na katangian nito. Ipinalalagay na ang wikang ito ay isang buhay na buhay na wika sa kasalukuyan na patuloy na umuunlad sa pamamagitan ng paggamit nito sa mabisang pagkakaunawaan at pagkakabuklod ng mamamayan ng mga Zamboangueño. Magbibigay linaw at magbubukas ito ng isipan sa ilang mga mamamayan na nagsasabi na ang wikang Zamboanga Chabacano ay walang sariling gramatikang kanya. Lalo na itinuturing ito na isang bastardized language (Camins, 1999).

Wikang Chabacano ang tawag sa wika ng lungsod ng Zamboanga. Ang wikang ito ay nagmula sa pidgin Spanish, subalit ito ay naging creole dahil sa proseso ng pinaghahalo-halo ng maraming wika kaya nabuo ang gramatikang Chabacano. Ang pidgin ay wikang nabuo o sumibol mula sa mga taong may iba't ibang wikang sinasalita. Kinakailangan nila ng isang wika na magiging instrumento sa kanilang pakikipagtalastasan at pakikipag-unawaan. Karaniwang nakabatay ang pidgin sa isa sa mga wikang sinasalita ng mga tao sa nasabing pook. Ginagamit na rin ito ng mga tao sa loob ng tahanan at sila ay nagkakaintindihan. Ayon pa kay Cuartocruz (1990), ang wikang Chabacano ay lumaganap sa Cavite, Ternate at Zamboanga. Binanggit naman ni Santiago (1979), nagmula sa wikang Espanyol ang wikang Chabacano at ang pinakalamalaking populasyon ng mga taong gumagamit ng wikang ito ay matatagpuan sa lungsod ng Zamboanga. Dagdag pa ni Frake (1990), ang wikang Chabacano ay hindi lamang isang wikang Filipino na may karaniwang mabigat na impluwensyang leksikal ng Espanyol, o isang Espanyol na may maraming bilang ng mga salitang hiram, ito ay natatanging wika na may natatanging paglalarawan sa gramatikal na kinabibilangan ng syntax, lexis, semantic at phonology.

May kaibahan ang naturang wika sa lengguwahe ng Kastila dahil magkaiba ang gramatika ng dalawang wika. Subalit, masasabing ang Zamboanga Chabacano ay Kastila dahil sa mga leksikon nito ngunit ang estruktura at gramatika nito ay ayon sa mga wikang katutubo ng Pilipinas. Sa katunayan, ang wikang Chabacano ay isa na sa itinuturing na pangunahing wikaing ginagamit sa buong lungsod ng Zamboanga sa kabila ng may iba't ibang wika pang ginagamit maliban sa wikang ito. Nagpapatunay lamang na ang wikang Chabacano bilang wika ng mga mamamayan ng lungsod Zamboanga ay mahalagang mabigyan ng sistematikong pag-aaral. Mula sa maraming kahalagahan na nabanggit, isinagawa ang pag-aaral na ito upang mabigyan ng tuon ang katangian ng estrukturang gramatikal ng wikang Chabacano.

\section{Metodolohiya}

Ginamit sa pag-aaral na ito ang disenyong kwalitatibo. Deskriptiv analisis naman ang ginamit sa pagsusuri ng wikang Zamboanga Chabacano. Inilarawan sa bahaging ito ang katangian ng pangungusap; bahagi, uri at ayos ng pangungusap ganoon din ang mga salitang pangkayarian at pagbuo ng pangungusap at ang pananda. 
Ginamit ang purposive sampling sa pagpili ng ng mga respondente sa pag-aaral na ito. Ang instrumento ng pag-aaral na ito ay mga aktuwal na usapan ng mga respondente. Ang mga usapang ito ay nagmula sa pitong domeyn; sa tahanan, sa paaralan, sa simbahan, sa terminal ng bus, sa palengke, sa internet Café at sa mall. Hindi pare-pareho ang bilang ng mga respondente sa bawat domain. Ang mga pangkat sa iba't ibang usapan ang siyang respondente ng pag-aaral na ito. Ang mga usapan sa pitong domeyn ay ginawa sa loob ng $\mathbf{7}$ kilometers radius mula sa City hall ng lungsod Zamboanga. Ang mga kasali sa usapan ay ay nasa pagitan ng labinlimang taong gulang hanggang animnapu at pataas. Upang matiyak na ang mga usapan ay sa wikang Chabacano, sinamahan ng isang taal na Zamboangueña ang mananaliksik sa pangangalap ng datos. matapos matiyak na ang usapan ay sa wikang Chabacano, sinimulan na ang pagrerekord ng usapan. Ito ay lingid sa mga respondente. Ang pagrerekord sa mga usapan ay ginawa sa loob ng sapung minuto at higit pa. Ang bilang ng mga respondenteng kasali sa usapan ay apat hanggang limang tao.

Pagkatapos na mairekord ang usapan, ito ay tinaranskrayb ng isang gurong taal na Zamboangueño at nagtuturo din sa Filipino. Inayos ng mananaliksik ang transkripsyon upang bigyan diin ang mga pangungusap, sinuri ang katangian ng pangungusap ayon sa bahagi, uri at ayos ng pangungusap. Binigyan pansin din sa pagsusuri ng mga pangungusap ang mga pang-ugnay at pananda sa loob ng pangungusap. Ang ginawang pagsusuri sa mga katangian ng pangungusap ng Zamboanga Chabacano ay binalideyt ng apat na gurong taal na Zamboangueño. Ang kanilang unang wika ay Zamboanga Chabacano at bihasa sa wikang Filipino. Ang mga nasabing balideytor ay may animnapu't limang gulang pataas. Lahat sila ay mga propesor din sa Filipino. Sa sintaktikal na pagsusuri pinag-aralan ang anyo at katangian ng iba't ibang pangungusap maging ang salitang pangkayarian at pananda sa loob ng pangungusap sa wikang Zamboanga Chabacano. Sa pag-aanalisa ng mga datos, ginamit ang mga aklat ni Paz et al. (2003). Naging kaagapay sa pagsusuri rin ang mga aklat at pag-aaral tungkol sa mga pangungusap nina Cruz (2005), Santos (2014), at Yap-Aizon (2010) sa wikang Zamboanga Chabacano.

\section{Paglalahad ng mga Resulta}

Sa bahaging ito pinag-aaralan ang gramar na may kinalaman sa sistema ng mga tuntunin at kategorya na siyang batayan sa pagbuo ng pangungusap sa wikang Zamboanga Chabacano. Taglay rin sa wikang Zamboanga Chabacano ang mga pangungusap na ganap at di-ganap. Ang paksa ay bahagi ng pangungusap na siyang pinagtutuunan ng pansin sa pangungusap. Sa paksa nakapukos ang diwa ng pangungusap. Maaaring ang paksa ng pangungusap ay tao, bagay, hayop, pook o pangyayari. Karaniwang ang paksa ng pangungusap ay may panandang pantukoy, madaling matukoy ang paksa ng isang pangungusap dahil sa pantukoy. Ang panaguri naman ay bahagi ng pangungusap na nagbibigay ng impormasyon tungkol sa paksa. Ito ang bahaging nagsasabi o naglalarawan tungkol sa paksa ng pangungusap, ang paksa at ang panaguri ay maaaring nasa unahan, gitna o hulihan ng pangungusap. Nakikilala ang paksa ng pangungusap sa wikang Zamboanga Chabacano sa pamamagitan ng pantukoy. Bukod sa panghalip, ang iba pang salita na kabilang sa mga salitang pangnilalaman na ginagamit bilang paksa ng pangungusap ay ginagamitan ng pantukoy na si para sa pangngalang pantangi at $\underline{e} \underline{l}$ para sa pangngalang pambalana, pandiwa at pang-uri. Ang $\underline{k a n d a}$ ay siyang anyong maramihan ng $\underline{\underline{i}}$ at $\underline{e l ~ m a g a}$ para sa anyong ng $\underline{e l}$. May iba't ibang uri ng ang paksa at panaguri ayon sa bahagi ng pananalita.

\section{Diskusyon}

\subsection{Paksa}

\section{Pariralang Pangngalan}

Halimbawang pangungusap

1. Ta enseña si maam Coreen. (Nagtuturo si maam Coreen).

Panaguri + Paksa 
Pahulaya, V. L.

Ta enseña + si maam Coreen

2. Plojo kanda Luis. (Tamad sina Luis.)

Panaguri + Paksa

Plojo + kanda Luis

3. Ya reclama el maga mayores. (Nagreklamo ang mga magulang.)

Panaguri + Paksa

Ya reclama + el maga mayores

Ipinakikita sa mga halimbawang pangungusap ang mga bahagi ng pangungusap. Mapapansin sa unang pangungusap ang paksa ay Maam Coreen, ginagamitan ng pantukoy na si. Sa ikalawang pangungusap ang paksa ay Luis, ito ay pinangungunahan ng pantukoy na kanda. Ito ang maramihan ng si, ang ikatlong pangungusap ang paksa ay mayores, ito naman ang pangngalang pambalana. May tiyak na pananda para sa pangngalang pantangi at pambalana. Ganoon din ang anyong isahan at maramihan ng nasabing pantukoy.

\section{Pariralang Panghalip}

1. Ya sale ele. (Umalis siya.)

panaguri + paksa

Ya sale ele

2. Con hambre ya yo. (Gutom na ako.)

panaguri + paksa

Con hambrea ya yo

3. Tiene Guerra alla. (May digmaan doon.)

Panaguri + Paksa

Tiene guerra alla

Makikita sa mga inilalahad na pangungusap na ang paksa sa mga ito ay mga panghalip. Sa unang pangungusap ang paksa ay panghalip na panao na ele, sa ikalawang pangungusap naman ay panghalip na panao na yo at sa ikatlong pangungusap ay panghalip na pamatlig na alla. Mapapansin na walang pantukoy ang mga panghalip na ginagamit sa pangungusap bilang paksa.

\section{Pariralang Pandiwa}

1. Su tata el ta corre. (Tatay niya ang tumakbo.)

panaguri + paksa

Su tata el ta corre

2. Vieja ya el ta laba plato. (Matanda na ang naghuhugas ng pinggan.)

panaguri + paksa

Vieja ya el ta laba plato

3. Iyo el limpia casa. (Ako ang maglilinis ng bahay.)

panaguri + paksa

Iyo el limpia casa

Ang mga paksa sa inilahad na mga pangungusap ay mga pariralang pandiwa. Ang pandiwa na ginagamit bilang paksa ay may panandang pantukoy na $e l$ na ang katumbas nito sa Filipino ay ang. Sa wikang Zamboanga

114 Consortia Academia Publishing (A partner of Network of Professional Researchers and Educators) 
Chabacano, ginagamitan ng pantukoy ang mga pandiwa na ginagamit sa pangungusap bilang paksa. Ang pandiwang corre at laba sa una at ikalawang pangungusap ay may panandang ta. Ito ay tumutukoy na ang pandiwa ay nasa aspektong imperpiktibo.

\section{Pariralang Pang-uri}

1. Ya perde el maga bonita. (Natalo ang mga magaganda.)

Panaguri + Paksa

Yaperde + el maga bonita

2. Ta ayuda na mga pobre el mga rico. (Tumutulong sa mga mahihirap ang mga mayayaman.)

Panaguri $+\quad$ Paksa

Ta ayuda na maga pobre + el maga rico

3. Hinde asensa el plojo. (Hindi aasenso ang tamad.)

Panaguri + Paksa

Hinde asensa + el plojo

Ang mga pariralang pang-uri sa mga inilahad sa pangungusap ay bonita, rico, at plojo. Ang mga ito ay may pandandang pantukoy na $e l$. Ang $e l$ ay ginagamit bilang pananda sa pang-uri na ginagamit sa pangungusap bilang paksa.

\section{Pariralang Pang-abay}

1. Copia tu el padetras. (Kopyahin mo ang patalikod.)

Panaguri + Paksa

Copiatu + el padetras

2. Limpia el adelante. (Linisin ang harapan.)

Panaguri + Paksa

Limpia + el adelante

3. Olvida ya el ayer. (Kalimutan ang kahapon.)

Panaguri + Paksa

Olvidaya + el ayer

Ang mga Pang-abay sa mga pangungusap na ginagamit bilang paksa ay padetras, adelante at ayer. Nakilala ang mga ito dahil sa pantukoy na $e l$ sa nasabing pangungusap.

\section{Pariralang Pang-ukol}

1. Tiene castigo el contra na ley. (May kaparusahan ang labag sa batas.)

Panaguri + Paksa

Tiene castigo + el contra na ley

2. Maga chinelas el para su anak.

(Mga tsinelas ang para sa kanyang anak.)

Panaguri + Paksa

Maga chinelas + el para su anak

3. Ya manda sale el maga andentro del KCC. 
Pahulaya, V. L.

(Pinaalis ang mga nasa loob ng KCC.)

Panaguri + Paksa

Ya manda sale + el maga adentro del KCC

Inilalahad sa mga pangungusap ang mga pariralang pang-ukol na ginagamit bilang paksa ng pangungusap. Ang halimbawang ito ay binubuo ng mga pang-ukol at pangngalan bilang layon ng pang-ukol. Sa unang pangungusap ang pang-ukol ay contra $n a$ at ang layon nito ay ang pangngalang ley. Sa ikalawang pangungusap ang pang-ukol ay para su at ang layon nito ang pangngalang anak. Sa huling pangungusap ang pang-ukol ay adentro del at ang layon ng pang-ukol na ito ay pangngalang Kcc.

Makikita sa mga inilalahad na halimbawa ng mga uri ng paksa ng mga pangungusap sa wikang Zamboanga Chabacano ay nagtataglay ng mga pantukoy. Nakikilala ang mga paksa ng pangungusap gamit ang mga pantukoy na si, el at ang anyong maramihan nito na kanda at el maga sa mga nasasbing pangungusap sa wikang Chabacano.

\subsection{Ang Panaguri}

Ito ay bahagi ng pangungusap na ganap na nagbibigay ng impormasyon tungkol sa paksa o naglalarawan sa paksa ng pangungusap. Ang karaniwang ayos ng pangungusap sa wikang Zamboanga Chabacano ay nauuna ang panaguri sa paksa. Inilalahad dito ang mga uri ng panaguri.

\section{Pariralang Pangngalan}

1. Maestra el mi nana. (Guro ang aking nanay.)

Panaguri + Paksa

Maestra + el mi nana

2. $\quad$ Si Tia Beng el gente na casa. (Si tiya Ben gang taong-bahay.)

Panaguri + Paksa

Si tia Beng + el gente na casa

3. Reporter ara si Kristine. (Reporter ngayon si Kristine.)

Panaguri + Paksa

Reporter ara + si Kristine

Ang mga panaguri sa mga nasabing pangungusap ay mga pangngalan. mapapansin na ang mga panaguring pangngalang pantangi ay may panandang si subalit ang mga pangngalang pambalana ay walang pananda o hindi ginagamitan ng panandang pantukoy.

\section{Pariralang Panghalip}

1. Ustedes el lleva comida. (Kayo ang magdadala ng pagkain.)

$\begin{array}{ll}\text { Panaguri } & + \text { Paksa } \\ \text { Ustedes } & + \text { el lleva comida }\end{array}$

2. Ese el tiene culpa. (Iyana ng may kasalanan.)

Panaguri + Paksa

Ese + el tiene culpa

3. Dimiyo el grande bag. (Akin ang malaking bag.)

Panaguri + Paksa

Dimiyo $\quad+\quad$ el granda bag

116 Consortia Academia Publishing (A partner of Network of Professional Researchers and Educators) 
Makikita sa mga halimbawang binanggit ang mga panghalip na ginagamit bilang panaguri ng pangungusap. Sa wikang Zamboanga Chabacano walang pananda ang mga panghalip na ginagamit sa pangungusap bilang panaguri man o paksa.

\section{Pariralang Pandiwa}

1. Ya munta bus el maga gente. (Sumaka ng bus ang mga tao.)

$\begin{array}{lll}\text { Panaguri } & + & \text { Paksa } \\ \text { Ya munta bus } & + & \text { el maga gente }\end{array}$

2. Ay anda na escuela el maga bata. (Pupunta sa paaralan ang mga bata.)

Panaguri + Paksa

Ay anda na escuela + el maga bata

3. Ta baña pa mi hermana. (Naliligo pa ang aking kapatid (babae).)

$\begin{array}{lll}\text { Panaguri } & + & \text { Paksa } \\ \text { Ta bañapa } & + & \text { mi hermana }\end{array}$

Makikita sa mga halimbawa ng mga pandiwa sa wikang Zamboanga Chabacano ng ginagamit bilang panaguri sa pangungusap. Ang mga pandiwa sa nasabing mga pangungusap ay may panandang $y a, \underline{a y}$ at $\underline{t a}$. Ang mga katagang ito ay nagbabadya ng aspekto ng pandiwa sa wikang Zamboanga Chabacano. Ang mga salitang-ugat na munta, anda at baña ay nasa anyong pawatas ng pandiwa. Walang impleksyon o hindi nagbabago ang anyo ng pandiwa. Tanging ang mga nasabing kataga lamang ang nagpapakita o nagsasabi ng aspekto ng pandiwa. Ang katagang ya para sa aspektong perpektibo, ang $\underline{a y}$ para sa aspektong kontempaltibo at $\underline{t a}$ para sa aspektong kontemplatibo.

Mapapansin din sa mga halimbawa ng panaguring pandiwa sa wikang Zamboanga Chabacano ay may layon o kaganapan ang pandiwa. Ang mga pandiwang ya munta ay may layong bus. Ang pandiwang anda ay may kaganapang ganapan. Subalit may mga pandiwa ring ginagamit bilang panaguri ang hindi nagtataglay ng layon o kaganapan. Tinatawag itong pandiwang katawanin.

\section{Pariralang Pang-uri}

1. Alto el di ila casa. (Mataas ang kanilang bahay.)

$$
\begin{array}{ll}
\text { Panaguri } & + \text { Paksa } \\
\text { Alto } & + \text { el di ila casa }
\end{array}
$$

2. Sabroso el potaje. (Masarap ang putahe.)

$$
\begin{aligned}
& \text { Panaguri }+ \text { Paksa } \\
& \text { Sabroso }+ \text { el potahe }
\end{aligned}
$$

3. Emfermosa si Anabel. (Sakitin si Anabel.)

$$
\begin{array}{ccc}
\text { Panaguri } & + & \text { Paksa } \\
\text { Emfermosa } & + & \text { si Anabel }
\end{array}
$$

Ipinakikita sa mga halimbawang ito ang mga pangungusap sa Zamboanga Chabacano na ginagamit ang pang-uri bilang panaguri. Walang pananda ang mga pang-uri bilang panaguri ng pangungusap. May panandang pantukoy ang mga pang-uri kapag ito ay ginagamit bilang paksa. 


\section{Pariralang Pang-abay}

1. Parriba el escribida di suyu nombre.

(Pataas ang pagsusulat ng kanyang pangalan.)

$$
\begin{array}{ll}
\text { Panaguri } & + \text { Paksa } \\
\text { Paariba } & + \text { el escribida di suyu nombre }
\end{array}
$$

2. Na Viernes el dalida del sueldo. (Sa Biyernes ang bigay ng sweldo.)

$$
\begin{aligned}
& \text { Panaguri }+ \text { Paksa } \\
& \text { Na Viernes }+ \text { el dalida sueldo }
\end{aligned}
$$

\section{Na lao derecha el entrada del casa.}

(Sa gawing kanan ang pasukan ng bahay.)

$$
\begin{aligned}
& \text { Panaguri }+ \text { Paksa } \\
& \text { Na lao derecho }+ \text { el entrada del casa }
\end{aligned}
$$

Ang mga panaguri sa mga halimbawang pangungusap ay mga pang-abay. Ang panaguring paariba ay pang-abay na pamaraan. Ang panaguri sa ikalawang pangungusap ay na Viernes. Ito ay nasa anyong pariralang pang-ukol ng ginagamit bilang pang-abay na pangungusap. Ito ay pang-abay na pamanahon. Ganoon din sa ikatlong pangungusap. Nasa anyong pariralang pang-ukol ang na lao derecho. Ito ay pang-abay na panlunan.

\section{Pariralang Pang-ukol}

1. Na mesa el maga plato. (Nasa mesa ang mga plato.)

$$
\begin{array}{lll}
\text { Panaguri } & + & \text { Paksa } \\
\text { Namesa } & + & \text { el maga plato }
\end{array}
$$

\section{Asegun na dueño el cosa ta hace kame.}

(Ayon sa may-ari ang ginagawa namin.)

$$
\begin{aligned}
& \text { Panaguri }+ \text { Paksa } \\
& \text { Asegun na dueño }+ \text { el cosa ta hace kame }
\end{aligned}
$$

\section{Para de ustedes el maga libro.}

$$
\begin{aligned}
& \text { Panaguri }+\quad \text { Paksa } \\
& \text { Para de ustedes }+\quad \text { el maga libro }
\end{aligned}
$$

Ang mga pariralang pang-ukol na ginagamit bilang panaguri sa pangungusap ay na mesa, asegun na dueño at para de ustedes. Ang pang-ukol na $\underline{n a}$ at $\underline{\text { asegun na }}$ ay mga layon ng para de ay isang panghalip. Ang mga pariralang pang-ukol sa wikang Zamboanga Chabacano ay binubuo ng pang-ukol at layon nito na maaaring pangngalan o panghalip.

\subsection{Ayos ng Pangungusap}

Taglay ng pangungusap ang dalawang bahagi; ang panaguri at ang paksa. Ang mga katutubong wika sa Pilipinas kasama na ang wikang Zamboanga Chabacano ay karaniwang ginagamit na nauuna ang panaguri sa paksa. Maging ang wikang Filipino ang karaniwang ayos ng pangungusap ay nauuna ang panaguri sa paksa. Subalit maaari ring mauna ang paksa sa pangungusap. Tinatawag itong di karaniwang ayos.

Karaniwang Ayos ng Pangungusap - Tinatawag na karaniwang ayos ng pangungusap sa wikang Zamboanga Chabacano kapag nauuna ang panaguri sa paksa. Mapapansin sa pakikipagtalastasan ng mga Zamboangueño laging nauuna ang panaguri sa paksa. Ito ay likas na ayos ng mga pangungusap sa wikang Chabacano. Bukod sa 
panghalip ang isang bahagi ng pananalita na ginagamit sa pangungusap bilang paksa ay laging may panandang pantukoy. Ito ang el, el maga, si at kanda. Ito ang mga halimbawa sa Filipino ng ang, ang mga, si at sina.

Mga halimbawa;

1. $\quad$ Ay gradua ele na Marso.

(Magtatapos siya sa Marso.)

2. Driver de bus el su tata.

(Driver ng bus ang kanyang tatay.)

3. Ta prepara ya si Mama el mesa.

(Naghahanda na ng mesa si Mama.)

4. Ya volbe ya kanda Emily.

(Umuwi na sina Emily.)

Ang karaniwang ayos ng pangungusap sa wikang Chabacano ay laging nauuna ang panaguri sa paksa. Ito ang malimit gamiting ayos ng pangungusap sa pormal man o di pormal na usapan. Ito ang likas na ayos ng pangungusap sa wikang ito. Nakikilala ang karaniwang ayos dahil nauuna ang panaguri at ang paksa naman ito ay laging may pananda maliban nga lamang kung ang paksa ay isang panghalip.

Di-Karaniwang Ayos na Pangungusap - Bagama't likas na anyo ang ng pangungusap sa wikang Zamboanga Chabacano na nauunaang panaguri sa paksa, katanggap-tanggap rin sa wikang ito na nauuna ang paksa sa panaguri. Ilipat man sa unahan ang paksa ng pangungusap, hindi pa rin nagbabago ang kahulugan ng nasabing pangungusap. Taglay pa rin ang paksa ang panandang pantukoy sa unahan man ito o sa hulihan. Kaya madaling makilala ang paksa dahil sa mga pantukoy.

Mga halimbawa;

1. Ele ay gradua na Marso.

(Siya ay magtatapos sa Marso.)

2. El su tata driver de bus.

(Ang kanyang tatay ay driver ng bus.)

3. Si Mama ta prepara ya el mesa.

(Si Mama ay naghahanda na ng mesa.)

4. Kanda Emily ya volbe ya.

(Sina Emily ay umuwi na.)

Ang mga pangungusap na nasa di-karaniwang ayos sa wikang Chabacano ay baliktad na maituturing sapagkat nauuna ang panaguri sa paksa. Dahil nga ang karaniwang ayos ay nauuna ang panaguri sa paksa. Sa kabila ng pagiging baliktad nito hindi nagbabago ang kahulugan at nanatili pa rin ang diwa ng mensahe sa nasabing pangungusap.

Sa wikang Filipino may pananda ang panaguri kapag ito ay nasa di-karaniwang aayos. Ito ang pangawing na ay. Hindi na ginagamit ang pangawing na ay kapag nauuna ang paksa sa panaguri. Kaya sinasabing ang ay ay pananda sa ayos ng pangungusap na nasa di-karaniwang ayos.

Magkatulad ang ayos ng pangungusap sa wikang Filipino ang ayos ng pangungusap sa wikang Zamboanga Chabacano. Karaniwang ayos, nauuna ang panaguri sa paksa at di-karaniwang ayos naman, nauuna ang paksa sa panaguri. Ang pagkakaiba nga lamang walang pananda ang panaguri sa wikang Chabacano maging karaniwan man o di-karaniwang ayos man. Ang ganitong ayos ng pangungusap sa wikang Chabacano ay sumasang-ayon sa 
pag-aaral ni Cruz (2005). Naaayon din ang natuklasan sa pag-aaral na ito sa pag-aaral ni Greenberg (1963), na binanggit niya ang konsepto tungkol sa language universals all language with VSO order have SVO as an alternative.

\subsection{Pagkakasunod-sunod ng mga salita sa Pangungusap}

Bukod sa maaaring mauna ang panaguri sa paksa o mauuna ang paksa sa panaguri sa isang pangungusap sa wikang Zamboanga Chabacano na hindi naman nagbabago ang kahulugan ng pangungusap, maari ding ilipat sa iba't ibang posisyon sa pangungusap ang iba pang komponent ng isang pangungusap. Hindi rin nagbabago ang kahulugan ng nasabing pangungusap.

Isa ito sa katangian ng mga pangungusap sa wikang Zamboanga Chabacano. Ang ibang komponent ng pangungusap ay naililipat sa unahan, gitna o hulihan ng pangungusap. Narito ang mga halimbawa;

\section{Karaniwang ayos:}

1. Ya compra el nana bag. (Bumili ang nanay ng bag.)

2. Ya compra bag el nana. (Bumili ng bag ang nanay.)

3. Bag ya compra el nana. (Bag bumili ang nanay.)

\section{Di-Karaniwang ayos:}

1. El nana ya compra bag. (Ang nanay ay bumili ng bag.)

2. El nana bag ya compra. * (Ang nanay bag ang bumili.)

3. Bag el nana ya compra * (Bag ang nanay bumili)

(Bag ang binili ng nanay.)

Ipinakikita sa mga halimbawa ng mga pangungusap sa wikang Chabacano ang iba't ibang posisyon ng komponent na layon ng pandiwa. Nasa karaniwang ayos o di-karaniwang ayos man ang pangungusap nailiipat ang layon ng pandiwa sa unahan, gitna at hulihan ng pangungusap na hindi nagbabago ang kahulugan. Ang ganitong katangian ng mga pangungusap sa wikang Chabacano ay inilahad din sa pag-aaral ni Yap-Aizon (2010).

Mapapansin din na ang mga layon ng pandiwa ay walang pananda. Sa Filipino ay laging may pananda ang layon ng pandiwa. Hindi maaaring ilipat sa ibang posisyon dahil magiging mali ang gramar nito o kinakailangang palitan ang anyo ng pandiwa upang maging tama ang gramar nito.

Ang karaniwang ayos ng pangungusap sa wikang Zamboanga Chabacano ay nauuna ang panaguri sa paksa. Ito ay natural na ayos ng kanyang pangungusap subalit maaari ding ipahayag ang ninais na nauuna ang paksa sa panaguri. Maging ang iba pang wikang katutubo na umiiiral sa Zamboanga Peninsula tulad ng Bisaya, Tausug, Yakan, at Subanon ay magkatulad din ang ayos ng pangungusap sa wikang Zamboanga Chabacano. Isang katangian ng wikang Zamboanga Chabacano na tanging ang paksa lamang bukod sa panghalip ay may pananda subalit ang panaguri ay walang pananda. Ang ilang komponent ng pangungusap tulad ng layon ng pandiwa ay naililipat sa unahan, gitna o hulihan ng pangungusap na hindi naman nagbabago ang kahulugan ng nasabing pangungusap.

Salitang Pangkayarian sa Pagbuo ng Pagungusap - Nahahati sa dalawang pangkat ang bahagi ng pananalita. ang una ay mga salitang pangnilalaman at ang ikalawa ay ang mga salitang pangkayarian. Ang mga salitang pangnilalaman ay mga salitang may mga tiyak na kahulugan. Ang mga ito ang nagsisilbing mahalagang salita sa loob ng pangungusap. Ang mga katangiang ito ng wika ay tunay na taglay rin sa wikang Chabacano. Iniuugnay nito ang iba't ibang uri ng salita na nakapaloob sa isang pahayag upang maging mas malinaw at buo ang diwa ng isang pangungusap.

Ang mga salitang pangkayarian naman ay mga salita o katagang walang tiyak na kahulugan kapag nag-iisa. 
Nagkakaroon lamang ng tiyak na kahulugan kapag ang mga ito ay ginagamit na sa pangungusap. Mahalagang papel ang ginagampamanan ng mga salitang pangkayarian sa pagbuo ng pangungusap. Nagiging malinaw at tiyak ang mensahe ng mga pangungusap. Ito ay maaaring nagtataglay ng paksa o panaguri sa iba't ibang anyo at uri ng pangungusap sa wikang Chabacano.

Nahahati naman sa dalawang subdibisyon ang mga salitang pangkayarian; (1) mga pang-ugnay at (2) mga pananda. Ang mga pag-ugnay salita o katagang nagpapakita ng kaugnayan ng dalawang yunit sa loob ng pangungusap o parirala. Samanatala, ang pananda naman ay nagsisilbing tanda ng gamit sa gramatikal ng isang salita sa pangungusap. Maaari ding ang mga pananda ay nagbabadya sa ayos ng pangungusap. Ginagamit ito bilang marker ng paksa at ng panaguri. Inilalahad sap ag-aarala na ito ang gamit ng mga pang-ugnay at pananda sa wikang Zamboanga Chabacano.

Ang mga salitang pangnilalaman ay nagtataglay ng sariling kahulugan kahit hindi gamitin sa pangungusap. Ang mga salitang pangkayarian ay wala o di-tiyak ang kahulugan kapag nag-iiba. Subalit lubhang mahalaga ang mga salitang pangkayarian sa pangungusap upang maging malinaw at mabisa ang anumang pahayag.

Mga Pang-ugnay - Ang mga pang-ugnay ay nabibilang sa mga uri ng salitang pangkayarian. Ang mga ito ay salita o katagang nagpapakita ng kaugnayan o relasyon ng isang salita, parirala o sugnay sa iba pang salita, parirala o sugnay sa loob ng pangungusap.

Ang pagkakaroon ng pagkakasunod-sunod ng mga pahayag o pangyayari sa loob ng pangungusap ay tunay na mahalaga, sapagkat naging mas makabuluhan ang pakikipag-ugnayan sa kapwa. May dalawang uri ng pang-ugnay ang Zamboanga Chabacano. Ito ay ang mga pangatnig at ang pang-ukol.

Pangatnig - Ang pangatnig ay maaaring kataga o salitang nag-uugnay ng dalawang salita, parirala o sugnay at pangungusap na pinagsusunod-sunod sa isang pangungusap. Ang mga ito ay nagpapalinaw at nagiging mabisa ang isang pangungusap.

May dalawang panlahat na pangkat ng mga pangatnig: (1) yaong nag-uugnay ng magkatimbang na yunit, (2) yaong nag-uugnay ng di magkatimbang na yunit. May mga pangatnig na ginagamit sa pag-uugnay ng mga salita ng wikang Chabacano bilang pangunahing wika na ginagamit sa pakikipagtalastasan.

Mga halimbawa:

1. Ta vende su nana na tiangge maga gulay, frutas $\boldsymbol{y}$ coco.

(Nagtitinda ang nanay niya sa palengke ng mga gulay, prutas at niyog)

2. Ya prepara ya ele el maga plato, baso pati maga cuchara.

(Inihanda na niya ang mga plato, baso at mga kutsara)

3. Cosa ba kamo hace aqui, compra maga ropa ontra sine?

(Ano ba ang gagawin ninyo rito, bumili ng mga damit o manood ng sine?)

4a. $\quad$ Si man buen bata ustedes, ay bendici con ustedes el Dios.

(Kung magiging mabuting bata kayo, pagpapalain kayo ng Diyos.)

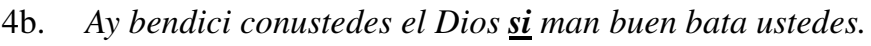

(Pagpapalain kayo ng Diyos kung magiging mabuting bata kayo

5. Quiere era yo sigui kaninyo pero tiene pa yo clase hasta alas cinco.

(Gusto ko sanang sumama sa inyo ngunit may klase pa ako hanggang alas singko.)

Ang una at ikalawang pangungusap ay gumagamit ng pangatnig na $y$ at pati na kapwa ang kahulugan sa Filipino ay at. Mapapansing iniuugnay ng mga ito ang mga salita, makikita ang mga pangatnig na ito bago ang huling salita na pinagsusunod-sunod sa pangungusap. Hindi ito ginagamit sa unahan ng pnagungusap. Ang 
pangatnig sa ikatlong pangungusap ay o. Ginagamit ito sa pag-uugnay ng dalawang parirala. Ang unang parirala ay compra maga ropa at ang pariralang entra cine. Ang pangatnig na ito ay hindi ginagamit sa unahan ng pangungusap. Sa ikaapat na pangungusap naman, iniuugnay ng pangatnig na $\underline{\boldsymbol{i}}$ ang isang punong sugnay at katulong na sugnay. Ang pangatnig na ito ay ginagamit sa katulong na sugnay. Mapapansin na ang pangatnig na $\underline{s}$ ay maaaring gamitin sa unahan ng pangungusap o sa huling bahagi. Ipinakikita rin dito na ang katulong na sugnay ang maaaring nasa unahan o hulihan ng punong sugnay. Ang ikalimang pangungusap naman ay isang tambalang pangungusap. Ang pangatnig dito ay pero. Nag-uuganay ito ng dalawang payak na pangungusap. Ang una ay "Quire yo sigui kaninyo" at ang ikalawa ay "Tiene pa yo klase hasta alas cinco"

Makikita sa mga halimbawang ito na ang mga pangatnig ng wikang Zamboanga Chabacano ay may kanya-kanyang tiyak na posisyon sa pangungusap. At ito ay hindi basta-basta na lang inililipat sa loob ng pangungusap. May mga pangatnig din na pwedeng ilipat ang posisyon ngunit isinasama ang sugnay na iniuugnay nito.

Mga halimbawa ng pangatnig sa wikang Zamboanga Chabacano:

1. Ya llega ya si Rosa, Helen y Teresa.

(Dumating na sina Rose, Helen at Teresa.)

2. Ta vende si Nora Paz maga gulay, frutas pati carne puerco.

(Nagtitinda ng mga gulay, prutas at karneng baboy si Aling Paz.)

3. El trabajo di Ester na casa cuci almuerzo, rega maga siembra y limpia casa.

(Ang gawain ni Ester sa bahay ay magluto ng almusal, magdilig ng halaman at maglinis ng bahay.)

4. Quiere era kame entra cine pero no hay kame sen.

(Gusto sana naming manood ng sine ngunit wala kaming pera.)

5. Ya puede acaba na studio su cinco maga anak maskin pobre lang sila.

(Nakapagtapos sap ag-aaral ang kanyang limang anak kahit mahirap lang sila.)

Sa una at ikatlong pangungusap ginamit ang pangatnig na $y$. ito ay katumbas ng at sa Filipino. Nag-uugnay ng mga salitang Helen at Teresa sa unang pangungusap. Sa ikatlong pangungusap naman, nag-uugnay ng mga pariralang rega maga siembra at limpia casa. Sa ikalawang pangungusap naman, ginamit ang pangatnig na pati na ang katumbas ay at pa rin sa Filipino. Nag-uugnay naman ito sa mga salitang frutas at carne puerco.

Ang sa ikaapat na pangungusap ang pangatnig ay pero. Ang katumbas nito sa Filipino ay ngunit. Iniugnay nito ang dalawang pangungusap na quiere era kame entra cine at no hay kame sen. Sa ikalimang pangungusap naman ang pangating ay maskin na ang katumbas nito sa Filipino ay kahit. Iniugnay nito ang dalawang sugnay na magkatimbag na ya puede acaba na studio si cinco maga anak at pobre lang sila. Ang pangatnig na pero at maskin ay nag-uuganay ng mga sugnay na magkatimbang.

Ang mga naunang mga halimbawa ay nagpapakita ng mga pangatnig ng Zamboanga Chabacano na nag-uugnay ng salita, at parirala. Ang mga pangatnig ay nauuri sa dalawang pangkat: yaong nag-uugnay na magkatimbang na yunit at yaong nag-uugnay ng di-magkatimbang na yunit. Ang mga pangatnig na nag-uugnay ng dalawa o higit pang sugnay na makapag-iisa. Karaniwan ang mga sugnay na nag-uugnay ng di- magkatimbang na yunit ay makikita sa unahan ng sugnay na di-makapag-isa.

Mga halimbawa:

1. $\quad$ Bien alboroto el maga estudiante mientras no hay pa el maestro.

(Napakaingay ng mga mag-aaral habang wala pa ang guro.)

2. Ay anda kame na pueblo pati ay compra kame diatom comida para medio dia. 
(Pupunta kami sa bayan at bibili kami ng ating pagkain para tanghalian.)

3. Ta enseña kanila si Jesus pero hinde sila ta cre.

(Nagtuturo sa kanila si Jesus ngunit hindi sila naniniwala.)

Ang mga pangatnig sa wikang Chabacano na inilalahad sa mga halimbawa ay nag-uugnay ng dalawang magkatimbang na payak na pangungusap o sugnay na makapag-iisa. Ang mga pangatnig sa pangungusap ang mientras, pati at pero.

Narito naman ang mga halimbawa ng mga pangatnig na nag-uugnay ng di-magkatimbang.

1. Yan sueldo ya tuyu tata entonces puede ya tu paga matricula.

(Nagsweldo na ang iyong tatay kung gayon maaari ka nang magbayad ng matrikula mo.)

2. Sigurao ay queda alegre tu anak si copmra tu ese bag.

(Tiyak magiging masaya ang iyong anak kung bibilhin mo iyang bag.)

3. Ya cuji con ele el pulis cay ya viola ele el ley.

(Himuli siya ng pulis dahil nilabag niya ang batas.

Ang mga pangatnig ng wikang Zamboanga Chabacano na entonces, si, at cay ay nag-ugnay ng punong sugnay at sugnay di-makapag-isa o katulong na sugnay. Ang pangungusap pagkapos ng mga pangatnig na ito ay siyang mga katulong na sugnay. Ang mga sumusunod na pangatnig ay ilan lamang sa iba pang mga halimbawa ng pangatnig sa wikang Chabacano. Ito ay mga nag-uugnay ng magkatimbang na yunit.

Pangatnig sa wikang Chabacano

$o$
$n i$
demas

mientras /mientras tanto

pero

y/pati

\section{Kahulugan}

$\mathrm{o}$

ni

at saka

habang/samantala

Ngunit/subalit

at

Mga pangatnig na nag-uugnay ng di magkatimbang na yunit.
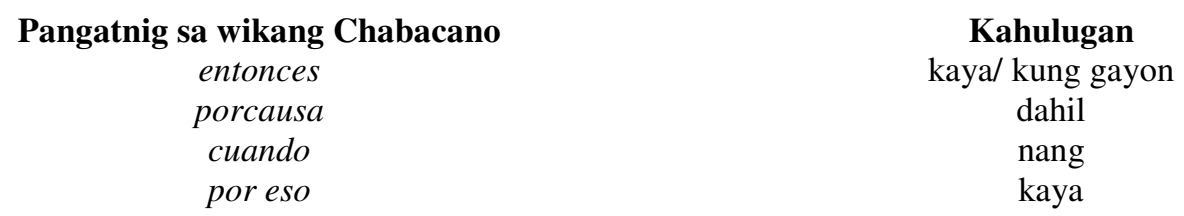

Ang ilan sa mga halimbawang pangatnig na ito ay mga salitang Kastila subalit ang iba ay hindi at maaaring hango ito sa ibang wikang katutubo ng Pilipinas.

Mga Pang-ukol - Isa sa mga pangkat na nabibilang sa pag-uugnay ay ang mga pang-ukol. Ito ay kataga o mga salitang nag-uugunay ng isang pangngalan o panghalip sa iba pang salita na pangungusap. Ito ay laging nauuna sa isang pangngalan o panghalip na nag-uugnay ang mga ito sa iba pang salita sa pangungusap. Taglay ng wikang Chabacano ang iba't ibang pang-ukol kung saan ito ay nagbibigay na malinaw sa isang pahayag. Pansinin ang mga halimbawa.

Mga halimbawa:

1. Para na maga anak el sacrificio del maga mayores.

(Para sa mga anak ang sakripisyo ng mga magulang)

2. Ya anda si Glenn para na escuela.

(Pumunta si Glenn para sa paaralan.) 
Pahulaya, V. L.

3. Ta llega el bus hasta conamon

(Dumadating ang bus hanggang sa amin.)

4. Recoje ya el maga gulay adentro del saco.

(Iligpit na sa sako ang mga gulay.)

5. De Zamboanga Sur y Norte el maga tindera na KCC.

(Taga-Zamboanga Sur at Norte ang mga tinder na KCC.)

Wikang Chabacano
$n a$
$d i$
$d e l$
cun
para na
para cun
asegun cun
kanda
asegun
hasta

Kahulugan sa Filipino
sa
ni
ng
kay
para sa
para kay
ayon kay
kina (maramihan ng cun)
naayon sa
hanggang

Ang mga pariralang pang-ukol ay makikita sa unahan, gitna o huling bahagi ng pangungusap. Sa unang pangungusap ang pangngalang sacrificio ay nag-uugnay sa pangngalang maga mayores. Sa ikalawang pangungusap naman, ang pangngalang Glenn ay nag-uugnay sa pangngalang escuela. Sa ikatlong pangungusap naman, ang pangngalang $\underline{\text { bus }}$ ay nag-uugnay sa panghalip na hasta. Sa ikaapat na pangungusap naman, ang pangngalang gulay ay nag-uugnay sa pangngalang saco. At sa ikalimang pangungusap naman, ang pangngalang $\underline{\text { tindera }}$ ay nag-uugnay sa pangngalang $\underline{K C C}$.

Ang mga pariralang pang-ukol ng wikang Chabacano ay binubuo ng pang-ukol at pangngalan o panghalip. Narito naman ang mga halimbawang pang-ukol sa loob ng pangungusap sa wikang Chabacano.

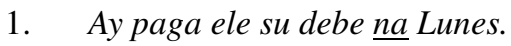

(Babayaran niya ang kanyang utang $\underline{\text { sa }}$ Lunes.

\section{Pabor arregla el maga flores na mesa}

(Pakiayos ang mga bulakluaak sa mesa.)

3. Ya dale yo cen cun Rica.

(Nagbigay ako ng pera kay Rica.)

4. $\quad$ Para de ustedes el maga guláy.

(Para sa inyo ang mga gúlay.)

5. Bien largo el pelo del dalaga.

(Napakahaba ang buhok ng dalaga.)

Sa mga halimbawang inilahad, ang pang-ukol sa una at ikalawang pangungusap ay na na may katumbas ito ng $\underline{\text { sa }}$ sa Filipino. Sa unang pangungusap, iniuugnay ng $\underline{n a}$ ang salitang Lunes at paga su debe. Sa ikalawang pangungusap naman iniuugnay ng $\underline{n a}$ ang salitang mesa at flores. Sa ikatlong pangungusap, ang pang-ukol ay $\underline{c u n}$ na ang katumbas nito sa Filipino ay kay. Iniuugnay nito ang salitang $\underline{\text { Rica }}$ at $\underline{\text { sen. }}$. Sa ikaapat na pangungusap, ang pang-ukol ay para de. Iniuugnay nito ang pangngalang gulay at panghalip na ustedes.

Ang katumbas sa Filipino ng pang-ukol na para de ay para sa. At sa huling pangungusap, ang pang-ukol ay $\underline{\mathrm{del}}$. Ang katumbas nito sa Filipino ay $\underline{n g}$. Iniuugnay nito ang salitang dalaga at pelo. Ang paggamit ng pang-ukol at pangngalan o panghalip ay bumubuo ng pariralang pang-ukol.

124 Consortia Academia Publishing (A partner of Network of Professional Researchers and Educators) 
The formation of Zamboanga Chabacano language sentences

Ang pinakaprominenteng pang-ukol sa Zamboanga Chabacano ay $n a$, de, di/del at cun. Ginagamit pa rin ang mga ito kasama ang iba pang pang-ukol.

Mapapansin na inuulit o ginagamit ang mga pang-ukol na na, cun, del/di/ de sa iba pang mga pang-ukol. Masasabi na ang mga pang-ukol ng wikang Zamboanga Chabacano ay hango o hiram sa wikang Kastila. Tunay nga na hindi maikakaila ang implementasyon ng wikang Kastila hindi lamang sa mga salitang pangnilalaman kung di maging sa mga pang-ugnay.

Patunay lamang na ang wikang Chabacano ay mayaman sa pang-ugnay na pangatnig at pang-ukol kung saan napakahalaga ang mga katagang ito sa loob ng pangungusap, dahil hindi maging malinaw ang mensaheng nais iparating kung wala ang mga ito. Ang wikang Chabacano ay may katangiang yunik na ikinaiiba sa ibang wikang katutubo ito ay ang pagiging creole na wika na pinagyaman mula sa iba't ibang wika ng Pilipinas.

Mga Pang-angkop - Ang isa pang uri ng mga pang-ugnay ay ang pang-angkop. Sa wikang Filipino ito ay katagang nag-uugnay sa panuring at salitang tinuturingan. Walang kahulugan ang mga pang-angkop. Napapaganda ang mga salita o pariralang pinaggagamitan nito. Nagiging maganda sa pandinig ang paggamit ng mga pang-angkop. Dalawa ang pang-angkop sa Filipino, ang na at ang ng. Iba ang distribusyon ng dalawang pang-angkop na ito. Pansinin ang mga sumusunod na halimbawa:

$\begin{array}{ll}\text { magandang dalaga } & \text { dalagang maganda } \\ \text { masipag na bata } & \text { batang masipag } \\ \text { halamang tuyo } & \text { tuyong halaman }\end{array}$

Sa wikang Filipino maaaring mauuna ang panuring o ang salitang tinuturingan. Ginagamit ang pang-angkop na na kung ang salitang_inaangkupan, panuring man o salitang tinuturingan ay nagtatapos sa katinig maliban sa letrang $\underline{\mathrm{n}}$. Kung nagtatapos naman sa $\underline{\mathrm{n}}$, nagkakaroon ng asimilasyon at naasimila ang $\underline{\mathrm{n}} \mathbf{s a} \underline{\mathrm{ng}} \underline{\mathrm{g}}$, at kapag nagtatapos naman sa patinig ang inaangkupan, ginagamit ang pang-angkop na ng.

Sa wikang Zamboanga Chabacano, hindi ginagamitan ng pang-angkop ang panuring at salitang tinuturingan. Pansinin ang mga sumusunod na halimbawa sa wikang Zamboanga Chabacano.

Pariralang Chabacano
bonito camisa
camisa bonito
camarin diutay
diutay camarin
layung ojas
ojas layung

Kahulugan
magandang damit
damit na maganda
kubong maliit
maliit na kubo
tuyong dahon
dahong tuyo

Makikita sa mga halimbawa ng mga parirala sa wikang Zamboanga Chabacano na wala o hindi gumagamit ng anumang pang-angkop o pang-ugnay sa pag-uugnay ng panuring at salitang tinuturingan. Ito ang likas na paraan ng pagpapahayag sa wikang Chabacano sa paglalarawan. Ang paraang ito ay katulad ng paraan sa wikang Kastila. Wala ring pang-angkop sa pag-uugnay ng panuring at salitang tinuturingan. Hango sa wikang Kastila ang hindi o walang pang-angkop, ang mga ibang wikang katutubo sa Pilipinas tulad ng Bisaya, kasama na ang Filipino ay may o gumagamit ng pang-angkop.

Dalawa lamang ang mga pang-ugnay sa wikang Zamboanga Chabacano. Ito ang pangatnig at pang-ukol. Ang mga pang-ugnay na ito ay karamihan hango sa wikang Kastila at ang ilan ay hango sa mga wikang katutubo sa Pilipinas. Ang paggamit ng mga pag-ugnay ay nagpapalinaw sa mensaheng nais iparating. Isang natatanging katangian ng wikang Zamboanga Chabacano ay maaaring mauna sa pangungusap o parirala ang panuring sa salitang tinuturingan o mauuna ang salitang tinuturingan sa panuring na hindi gumagamit o walang pang-ugnay na pang-angkop. Ang ganitong estruktura ay hango o impluwensya ng wikang Kastila.

Mga Pananda - Ang mga pananda ay nagsisilbing tanda ng gamit na panggramatikal ng isang salita sa loob ng 
pangungusap. Maaari ding ang mga pananda ay nagbabadya ng paksa o panaguri ng pangungusap. Sa pamamagitan ng pananda nakikilala rin ang ayos ng pangungusap, kung ito ba ay nasa karaniwan o di-karaniwang ayos ang nasabing pangungusap.

Ang wikang Zamboanga Chabacano ay may tatlong uri ng pananda; (1) ang pantukoy (2) ang pang-ukol bilang pananda sa pangngalang ginagamit bilang panuring, (3) mga kataga bilang pananda sa aspektong pandiwa. Ang mga pantukoy at mga pang-ukol na ginagamit bilang pananda sa gamit ng pangngalan o panghalip sa pangungusap ay may anyong kailanang isahan at paramihan.

Ang mga Pantukoy ng Wikang Chabacano - Ang mga pantukoy ay katagang laging nangunguna sa pangngalan o panghalip na ginagamit bilang simuno o kaganapang pansimuno sa pangungusap bilang paksa, panaguri o maaaring pamuno sa mga ito. Ang pantukoy ay pananda ng gamit sa palagyo ng pangngalan o panghalip. Sa kahulugang pansemantika, masasabing ang mga pantukoy ay kataga o mga katagang lumilinaw sa kailanan ng kasamang pangngalan o panghalip at kung ito ay tumutukoy sa tao, bagay o pook. Sa wikang Filipino, ang mga pananda ng pambalarilang gamit ng isang salita ang si at sina ay mga pantukoy sa pantanging ngalan ng tao. Ang at ang mga ay mga pantukoy sa pangngalang pambalana at pangngalang pantanging ngalan ng pook o

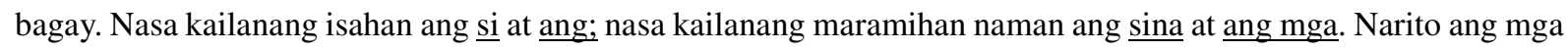
pantukoy ng wikang Chabacano.

Wikang Chabacano
si
kanda
el
el maga

Katumbas sa Filipino
si
sina
ang
ang mga

Ang mga pantukoy ng wikang Zamboanga Chabacano ay el at el maga, para sa pananda ng pangngalan o panghalip sa kailanang isahan at maramihan. Ang mga ito ay ginagamit bilang pananda sa pangngalang pambalana. Ang pantukoy na $s i$ at kanda ay pananda sa mga pangngalang pantangi para sa kailanang isahan at maramihan. Pansinin ang mga halimbawa ng pantukoy bilang pananda sa pangngalan o panghalip sa kaukulang palagyo:

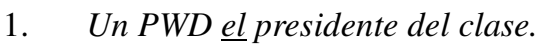

(Isang PWD ang pangulo ng klase.)

2. De Sibugay el maga tindera del nuevo mall.

(Taga Sibugay ang mga tindera ng bagong mall.)

3. Ta ayuda el dalaga cun Isabel limpia el cuarto.

(Tumutulong ang dalaga kay Isabel sa paglilinis ng kwarto.)

4. Ya llega con hambre na escuela kanda Susan, Dina y Jane.

(Dumating na gutom sa paaralan sina Susan, Dina at Jane.)

5. Talla pa na faculty room kanda Maam Ditas.

(Naroon pa sa faculty room sina Maam Ditas.)

6. Saca ya tu el di aton.

(Kunin mo na ang atin.)

Ipinakikita sa mga halimbawang pangungusap ang mga pantukoy sa wikang Chabacano. Ang mga pantukoy na $\underline{e l}$ at $\underline{e l ~ m a g a}$ ay ginagamit bilang pananda sa pangngalang pambalana na ginagamit sa pangungusap bilang paksa. Ang $e l$ ay kailanang isahan at ang el maga ay kailanang maramihan. Ang pantukoy na ginagamit bilang pananda sa mga pantangi ay $\underline{s i}$ para sa kailanang isahan at kanda para sa kailanang maramihan. Ang pantukoy na si sa ikatlong pangungusap ay ginagamit bilang pananda sa pamuno sa paksa o simuno. Mapapansin din sa ikalimang halimbawang pangungusap ang pantukoy na kanda ay para sa kailanang maramihan subalit sa pangungusap na ito 
isang pangngalang pantangi lamang ang sumusunod dito. Gayon pa man maramihan pa rin ang tinatalakay nito dahil ang ibig sabihin nito si Maam Ditas at ang kanyang mga kasama, maaaring mga guro din o ibang tao. Ang ikaanim na pangungusap ay gumagamit ng pantukoy na $e l$ bilang pananda sa panghalip na gingamit bilang paksa ng pangungusap.

Ang Pang-ukol Bilang Pananda sa Pangngalan at Panghalip na Ginagamit sa Pangungusap sa Kaukulang Palayon at Paari - Ang pang-ukol bagama't isa sa mga pang-ugnay dahil nagpapakita ito ng kaugnayan ng isang pangngalan o panghalip sa isa pang salita sa pangungusap ay ginagamit din bilang pananda sa pangngalan at panghalip sa kaukulang palayon at paari. Ang mga pang-ukol na ito na ginagamit bilang pananda ay may anyo rin para sa kailanang isahan at maramihan gayon din para sa pananda sa pangngalang pambalana at pantangi.

Mga halimbawang pangungusap:

1. Ya confisca el licencia del driver.

(Kunumpiska ang lisensya ng driver.)

2. Ta pensa el maga mayores el futuro del maga anak.

(Iniisip ng mga magulang ang kinabukasan ng kanilang mga anak.)

3. Alto el respeto del maga empleado con el maneger.

(Mataas ang respeto ng mga empleyado sa manager.)

4. Debe ba kita cre con el maga promesa diila?

Dapat ba tayong maniniwala sa mga pangako nila?)

5. Grande pa el esperanza di Ňora Tess.

(Malaki pa ang pag-asa ni Aling Tess.)

6. Alto-alto el maga grado di kanda Katrina y su maga amega.

(Matataas ang mga grado nina Katrina at ng kanyang mga kaibigan.)

7. Ta ayuda cun Kapitan Alberto el maga kagawad del barangay.

(Tumutulong kay Kapitan Alberto ang mga kagawad ng barangay.)

8. Ya habla le siempre el verdad cun kanda Jennefer.

(Sinabi pa rin niya ang katotohanan kina Jennefer.)

Ipinakikita sa mga maraming pangungusap na halimbawa ang iba't ibang pang-ukol sa wikang Zamboanga Chabacano na ginagamit sa pangungusap bilang mga pananda sa pangngalan at panghalip sa kaukulang palayon at paari. Ang pang-ukol sa unang pangungusap ay $\underline{\mathrm{del}}$, ito ay may kailanang isahan at ginagamit sa pangngalang pambalana. Sa ikalawang pangungusap del maga para sa kailanang maramihan, kapwa nasa kaukulang paari ang mga pang-ukol na ito. Ang ikatlo at ikaapat na pangungusap ay nagpapakita ng mga pang-ukol bilang pananda sa mga pangngalang nasa kaukulang palayon. Ang pang-ukol na $\underline{\text { cun }}$ ay nasa kailanang isahan at ang anyong maramihan naman nito ay cun el maga. Ginagamit pa rin ang mga ito para sa pangngalang pambalana. Ang mga pang-ukol sa ikalima, ikaanim, ikapito at ikawalong pangungusap ay ginagamit para sa tanging pangngalan. Ang pang-ukol na $\underline{d i}$ ay nasa anyong isahan at ang anyong maramihan nito ay di kanda. Ang mga ito ay pananda sa pangngalang nasa ikalawang paari. Ang mga pang-ukol naman sa ikapito at ikaanim na pangungusap ay $\underline{\text { cun }}$ para sa isahan at cun kanda naman para sa kaulukang maramihan. Ang mga pang-ukol na ito ay pananda sa pangngalang ginagamit sa kaukulang palayon.

Tunghayan ang kabuuang mga pananda sa panggalang nasa kaukulang palagyo, paari at palayon. 
Pahulaya, V. L.

\section{Talahanayan 1}

Kabuuang Pananda sa Pangngalang Palagyo, Paari at Palayon

\begin{tabular}{|c|c|c|c|c|c|c|c|c|}
\hline \multirow{3}{*}{$\begin{array}{c}\text { Kaukulan } \\
\text { Pananda ng } \\
\text { pangngalan }\end{array}$} & \multicolumn{4}{|c|}{ Pangngalang Pambalana } & \multicolumn{4}{|c|}{ Pangngalang Pantangi } \\
\hline & \multicolumn{2}{|c|}{ Isahan } & \multicolumn{2}{|c|}{ Maramihan } & \multicolumn{2}{|c|}{ Isahan } & \multicolumn{2}{|c|}{ Maramihan } \\
\hline & $\begin{array}{c}\text { Chabacan } \\
o\end{array}$ & Fil & $\begin{array}{c}\text { Chabacan } \\
o\end{array}$ & Fil & Chabacano & Fil & $\begin{array}{c}\text { Chabacan } \\
o\end{array}$ & Fil \\
\hline Palagyo & el & ang & el maga & $\begin{array}{l}\text { ang } \\
\text { mga }\end{array}$ & si & si & kanda & sina \\
\hline $\begin{array}{c}\text { Paari } \\
\text { Palayon }\end{array}$ & $\begin{array}{c}\text { del } \\
\text { cun el }\end{array}$ & $\begin{array}{l}\text { ng } \\
\text { sa }\end{array}$ & $\begin{array}{l}\text { del maga } \\
\text { cun el } \\
\text { maga }\end{array}$ & $\begin{array}{l}\text { ng mga } \\
\text { sa mga }\end{array}$ & $\begin{array}{c}d i \\
\text { cun }\end{array}$ & $\begin{array}{c}\text { ni } \\
\text { kay }\end{array}$ & $\begin{array}{c}\text { di kanda } \\
\text { cun kanda }\end{array}$ & $\begin{array}{l}\text { nina } \\
\text { kina }\end{array}$ \\
\hline
\end{tabular}

Mapapansin sa mga pananda sa pangngalan ng wikang Zamboanga Chabacano ang impluwensya o hango sa wikang Kastila. Ang mga ito ay pantukoy na $\underline{e l}$ para sa kaukulang palagyo sa kailanang isahan at ang si para sa tanging ngalan. Ang pang-ukol na $\underline{c u n} \mathrm{o} \underline{c o n}$, ang ilang Zamboangueño ay binibigkas ng $\underline{c u n}$ ang iba naman ay con, alinman sa dalawa ay tinatanggap ito. Bukod sa mga binanggit ang iba pang pantukoy ay likas na wikang Chabacano o maaring hango sa ibang wikang katutubo sa Pilipinas.

Ang mga pananda para sa pangngalang pambalana sa kaukulang isahan ay $\underline{e l}$ para sa kaukulang palagyo, $\underline{\mathrm{del}}$ para sa kaukulang paari at cun el para sa kaukulang palayon. Napakapayak at simple lamang ang pagbuo ng anyong maramihan ng mga panandang ito. Ito ay sa pamamagitan ng paggamit ng isahan at saka idagdag o gamitin ang salitang maga. Ginagamit ang salitang ito sa lahat ng kaukulan upang ipahayag ang kailanang maramihan. Kaya ang anyong isahan ay el ang maramihan ay el maga, ang isahan ay del, ang maramihan ay del maga at isahan ay cun el ang anyong maramihan naman ay cun el maga.

Para naman sa mga pananda sa pangngalang pantangi sa kailanang isahan ginagamit ang pantukoy na si para sa kaukulang palagyo. Ginagamit ang pang-ukol na di para sa kaukulang paari at cun para sa kaukulang palayon. Gaya sa mga pananda sa pangngalang pambalana iisang salita ang ginagamit para sa pagtukoy ng maramihan sa iba't ibang kaukulan, kung sa pangngalang pambalana ang anyong maramihan ay paggamit ng salitang maga, sa pangngalang pantangi naman ay kanda. Sa kaukulang palagyo ang maramihan ay kanda, sa kaukulang paari ay anyong isahang $d i$ at ang maramihang kanda kaya di kanda. Sa kaukulang palayon naman ang isahan ay $\underline{\text { cun }}$ ang maramihan ay cun kanda.

Mga Pananda sa Aspekto ng Pandiwa sa Wikang Chabacano - Isa sa mga natatanging katangian ng wikang Chabacano ay ang iba't ibang aspekto ng pandiwa. Walang impleksyon o hindi nagbabago ang anyo ng pandiwa ayon sa iba't ibang aspekto. Wala ring anuman anyong pawatas ang mga pandiwa sa wikang ito. Ang mga pandiwa ay nasa anyo o kayariang payak. May mga tiyak na pananda ang ginagamit upang ipahayag ang tatlong aspekto ng pandiwa. Ang mga ito ay ang mga katangang ya, ta at $a y$.

Ang katagang ya ay ginagamit bilang panada sa aspekto ng pandiwa na perpektibo. Ang kilos ay nasimulan at tapos na.

Mga halimbawa:

1. Ya entra kame cine ayer.

(Nanood kami ng sine kahapon.)

2. Ya volve el Tio na Labuan.

(Umuwi ang tiyuhin sa Labuan.)

3. Ya pensa ele enbuenamente.

(Nag-isip siya nang maayos.)

128 Consortia Academia Publishing (A partner of Network of Professional Researchers and Educators) 
Mapapansin sa mga halimbawa na ang anyo ng pandiwa ay payak o salitang-ugat lamang. Ang katagang ya bago ang salitang-ugat ay nagbabadya na ang kilos ng pandiwa ay tapos na mayroon man o walang salitang nagsasaad ng panahon.

Ang katagang $t a$ ay nagsisilbing pananda sa pandiwang nasa aspektong imperpektibo. Ang kilos ay nasimulan na at patuloy pang nagaganap at hindi pa tapos. Maaari ding kilos ay palaging ginagawa.

Mga halimbawa:

1. Ta munta ele jeep si ta anda le na escuela.

(Sumasakay siya ng dyip kung pumupunta siya sa paaralan.)

2. Ta toma ele leche todo el dia.

(Umiinom siya ng gatas araw-araw.)

3. Ta busca pa trabajo si Rommel.

(Naghahanap pa ng trabaho si Rommel.)

Makikita sa mga salin o katumbas sa Filipino ang anyo ng pandiwa ay nasa aspektong imperpektibo. Sa Zamboanga Chabacano naman matutukoy ang aspekto dahil sa panandang $t a$.

Ang katagang ay naman ay pananda sa pandiwang nasa aspektong kontemplatibo. Ang kilos ng pandiwa sa aspektong ito ay hindi pa nagaganap. Mangyayari pa lamang ang kilos.

Mga halimbawa:

1. Ay busca ya lang kita ayudante para aqui na casa.

(Maghahanap na lang tayo ng katulong para ditto sa bahay.)

2. Ay espera yo cun ustedes na Domingo.

(Hihintayin ko kayo sa Linggo.)

3. Ay sufri tu si casa tu cunese hombre.

(Magdurusa ka kung pakakasalan mo ang lalaking iyan.)

Ang kilos ng pandiwa sa mga halimbawa sa pangungusap ay nagsasaad na ang kilos ay hindi pa nagaganap. Sa unang pangungusap bagama't walang salitang nagsasaad ng panahon, natutukoy na ang kilos ang magaganap o mangyayari pa lamang dahil ginamit o may panandang ay na nagbabadya na ang kilos ay mangyayari pa lamang. Sa ikalawang pangungusap naman may pahayag o parirala na pangungusap naman may pahayag o parirala na nagsasabi ng panahon, na Domingo. Ang pahayag na ito ay nangangahulugan na hindi pa nangyayari ang kilos kaya ginamit ang panandang $\underline{a y}$.

Nagiging malinaw at tiyak ang mensahe ng mga pangungusap sa wikang Zamboanga Chabacano sa tulong o gamit ng pangngalan na pangungusap dahil sa mga pananda.

Madaling matukoy at maipahayag sa wikang Zamboanga Chabacano ang iba't ibang aspekto ng pandiwa sa pamamagitan lamang ng mga katagang $y a$, ta at $a y$.

Ang mga pananda sa gamit ng pangngalan sa pangungusap at pananda sa aspekto ng pandiwa ay napakasimple at madaling matukoy at magamit. Sa ganitong katangian ng wikang Zamboanga Chabacano masasabing madali lamang matutunan ang wikang ito.

\section{Kongklusyon}

Wika ang ginagamit ng tao sa pakikipagtalastsasan. Naipapaabot niya sa kanyang kapwa ang nais niyang iparating ang anumang mensahe gamit ang kanyang wika. Masasabing ang pinakadakilang handog ng Diyos sa tao 
ay ang wika, kung pagbabatayan ang divine origin of language. Wika ang ginagamit ng tao sa kanyang pakikipag-ugnayan at pakikihalubilo sa mga taong kabilang sa kanyang lipunan.

Lahat ng wika ay may gramar. Katulad ng wikang Zamboanga Chabacano na nagsimula sa pagiging pidgin at nang lumaon ay naging wika ng tahanan na mga mamamayan ng Zamboanga ay nag-aangkin din ng gramar na katulad sa ibang wikang katutubo at iba pang wika. Ang wikang Zamboanga Chabacano ay nagtataglay rin ng gramatikal na katangiang may malinaw na sistemang pangwika.

Likas sa ayos ng pangungusap ng Zamboanga Chabacano ang nauuna ang panaguri sa paksa subalit maaari ding nauuna ang paksa sa panaguri. Ganito man ang ayos ng pangungusap, hindi nagbabago ang kahulugan ng nasabing pangungusap. May pananda ang paksa ng pangungusap subalit wala o hindi gumagamit ng pananda ang panaguri. Ang mga kategoryang sintaktikal na ginagamit bilang paksa ay ginagamit din bilang panaguri. Ang wikang ito ay nagtataglay ng mga salitang pangkayarian na ginagamit sa pagbubuo ng mga pangungusap. Nagiging malinaw at ganap ang kahulugan ng mga pangungusap sa pamamagitan ng mga pangatnig, pang-ukol at mga pananda. Isang kaakuan ng mga pag-ugnay ng wikang Zamboanga Chabacano ay gumaganap din sa tungkulin ng pananda. Ang pang-ukol ay ginagamit na pang-ugnay subalit maari din itong gumanap na tungkulin ng pananda sa pangngalan sa loob ng pangungusap.

Madaling makilala ang paksang pangungusap sa wikang Zamboanga Chabacano dahil may pananda ito sa loob ng pangungusap maliban sa panghalip na nasa anyong palagyo. Hindi gaanong mahigpit ang pagtakda ng pagkakasunod -sunod ng mga salitang pangninlalaman ng pangungusap. Maaaring ilipat sa iba't ibang posisyon; unahan, gitna o hulihan. Subalit ang mga salitang pangkayarian ay may tiyak na posisyon sa pangungusap ayon sa tungkulin nito. Hindi rin gumagamit o walang pang-angkop sa wikang Zamboanga Chabacano. Ang bakas ng wikang Espanyol at maging ng wikang katutubo sa wikang ito ay nasa kanyang leksikon. Ganoon pa man, ang mga pangungusap ng Zamboanga Chabacano ay nagtataglay ng kanyang sariling kaakuan na hindi Kastila o wikang katutubo.

\subsection{Rekomendasyon}

Napapanahon ang pag-aaral na ito dahil nakatutulong ito bilang karagdagang sanggunian at kagamitan ng mga guro sa pagpapatupad sa programa ng Deped ayon sa Kautusang Pangkawaran blg.16, S. 2012. Ito ay mga panuntunan sa pagpapatupad ng Mother Tongue Based - Multilingual Education (MTB_MLE). Ginagamit at itinuturo ang unang wika ng mga mag-aaral mula sa kindergarten hanggang sa Grade 3. Sa lungsod ng Zamboanga bagama't may iba't ibang wika sa tahanan ang mga bata, may paraang ipinatutupad ng programa ito ng Deped.

Iminumungkahi rin na ang resulta na ito ay ilalagak sa aklatan ng lungsod ng Zamboanga nang sa ganoon magiging karagdagan materyal para sa nagnanais ng mga mag-aaral tungkol sa wikang Zambonga Chabacano. Ang resulta ng pag-aaral na ito ay hindi lamang para sa mga gurong nagtuturo o sangkot sa MTB-MLE na programa ng Deped kung di sa lahat ng guro o sinuman na gustong mag-aral at matutu ng wikang Zamboanga Chabacano.

\section{Mga Sanggunian}

Bucjan, E.R. (2017). Word formation in the kamayo language: A morphosyntactict analysis [Pagbuo ng mga salita sa wikang Kamayo: Isang morposintaktikal na pagsusuri]. Disertasyon. MSU-Iligan Institute of Technology. Iligan City.

Bucoy, C. B. (1985). The english and the chabacano basic statement patern: A comparative analysis. Zamboanga City: WMSU.

Camins, B. S. (1999). Chabacano de Zamboanga handbook. Philippine, Claretian Publishing.

Cantillo, Ma. L. M. Gime, A.V., \& Gonzales, A. P. (2015). Communication and research in the Filipino language and culture [Komunikasyon at pananaliksik sa wika at kulturang Pilipino.] Quezon City: St. Bernadette Publishing House Corporation. 
Carter, R. (2008), Pedagogical grammar: Glossary of Grammatical and rhetorical terms. Retrieved april 20, 2018 from http://grammar.about.com/od/pq/g/

Chomsky, N. (1964). Current issues in linguistic theory. The Hague: Mouton \& Co.

Chomsky, N. (1965). Syntactic structures. The Hague: Mouton \& Co.

Cruz, L. C. (2005). The syntax of Zamboanga Chabacano: a desriptio and analysis. Zamboanga City: WMSU.

De Castro, I. P. et al. (2007). Filipino language in academic communication [Wikang filipino sa akademikong komunikasyon.] Mutya Publishing House, Inc, Valenzuela City.

Edding, N. R. (2003). Language maintenance and shift among native and non- native speakers of zamboanga Chabacano. Zamboanga City: WMSU.

Fromklin, V. et al. (2003). An introduction to language ( $7^{\text {th }}$ ed.). Thomson: Heinle.

Greenberg, J. H. (1966). Universals of language ( $2^{\text {nd }}$ ed.). Cambridge: The M.I.T. Press.

Hudson, R. A. (2004). Why education needs linguistics (and vice versa). Journal of Linguuistics, 40, 105-130. https://doi.org/10.1017/S0022226703002342

Ladefoged, P. (1971). Preliminaries to linguistic phonetics. Chicago: University of Chicago Press.

Mangaser, V. D., \& Vilaneza, R. (2012), Bene y le, guria para na enseñanza-primer grado. IMCS Pepartment of Education Pasig, Metro Manila.

O’ Grady, W. O., \& Dobrovolsky, E. (1992). Contemporary linguistic analysis. An entroduction (2 ${ }^{\text {nd }}$ ed.). Toronto: Copp clark Pitman Ltd. A longman Company.

O'Grady, W., \& Archibald, J. (2004). Contemporary linguistics analysis (5 ${ }^{\text {th }}$ ed.) Ontario: Pearson Education Canada Inc.

Paz, C. J. (2003), Study of language [Ang pag-aaral ng wika.] Quezon City: The University of the Philippines Press.

Real, A. S. (2018), Grammar reference of the teduray language [Reperensyang gramatika ng wikang Teduray.] Iligan City: MSU-IIT.

Samarca, Y. A. (2016). Descriptive study of the grammatical structure of the cantilangnun language in surigao del norte. [Deskriptibong pag-aaral ng estrukturang gramatikal ng wikang Cantilangnun sa Surigao del Sur.] Iligan City: MSU-IIT.

Santiago, A. O., \& Tiangco, N. G. (1991), Modern filipino grammar [Makabagong balarilang filipino.] Rex Printing Company, Inc: Quezon City.

Santiago, A. O., \& Tiangco, N. G. (2003), Modern filipino grammar [Makabagong balarilang filipino.] Rex Book Store, Metro Manila.

Santos, R. A. (2014), Chabacano de zamboanga compedenio y dicionario. Zamboanga City: Ateneo de Zamboanga University Press.

Simpson, S. A. (1979). A first course in linguistics. Edinburgh: Edinburgh University Publication, Inc. Yap-Aizon, J. G. (2010). Zamboanga Chabacano: Structure and grammar. Manila: Adamson University Press. Yap-Aizon, J. G. et al. (2016). Riquezas: Chavacano essays and studies: Zamboanga City: Local government of Zamboanga. 
Pahulaya, V. L.

132 Consortia Academia Publishing (A partner of Network of Professional Researchers and Educators) 